\title{
Processed red meat contribution to dietary patterns and the associated cardio-metabolic outcomes
}

\author{
Yvonne M. Lenighan ${ }^{1}$, Anne P. Nugent ${ }^{2}$, Kaifeng F. Li ${ }^{2}$, Lorraine Brennan ${ }^{2}$, Janette Walton ${ }^{3}$, \\ Albert Flynn ${ }^{3}$, Helen M. Roche ${ }^{1}$ and Breige A. McNulty ${ }^{2 *}$ \\ ${ }^{1}$ Nutrigenomics Research Group, School of Public Health, Physiotherapy and Sports Science, UCD Conway Institute, University \\ College Dublin, Belfield, Dublin 4, Republic of Ireland \\ ${ }^{2}$ School of Agriculture and Food Science, UCD Institute of Food and Health, University College Dublin, Belfield, Dublin 4, Republic \\ of Ireland \\ ${ }^{3}$ School of Food and Nutritional Sciences, University College Cork, Cork, Republic of Ireland \\ (Submitted 15 March 2017 - Final revision received 19 June 2017 - Accepted 12 July 2017)
}

\section{Abstract}

Evidence suggests that processed red meat consumption is a risk factor for CVD and type 2 diabetes (T2D). This analysis investigates the association between dietary patterns, their processed red meat contributions, and association with blood biomarkers of CVD and T2D, in 786 Irish adults (18-90 years) using cross-sectional data from a 2011 national food consumption survey. All meat-containing foods consumed were assigned to four food groups $(n$ 502) on the basis of whether they contained red or white meat and whether they were processed or unprocessed. The remaining foods ( $n$ 2050) were assigned to twenty-nine food groups. Two-step and $k$-means cluster analyses were applied to derive dietary patterns. Nutrient intakes, plasma fatty acids and biomarkers of CVD and T2D were assessed. A total of four dietary patterns were derived. In comparison with the pattern with lower contributions from processed red meat, the dietary pattern with greater processed red meat intakes presented a poorer Alternate Healthy Eating Index (21.2 (SD 7.7)), a greater proportion of smokers (29\%) and lower plasma EPA (1.34 (SD 0.72)\%) and DHA (2.21 (sD 0.84)\%) levels $(P<0.001)$. There were no differences in classical biomarkers of CVD and T2D, including serum cholesterol and insulin, across dietary patterns. This suggests that the consideration of processed red meat consumption as a risk factor for CVD and T2D may need to be re-assessed.

\section{Key words: Processed red meat: CVD: Type 2 diabetes: Dietary pattern analysis}

Consumption of red meat has been associated with the development of chronic diseases including the metabolic syndrome (MetS), CVD and type 2 diabetes (T2D) ${ }^{(1,2)}$. With the prevalence of these diseases increasing rapidly, the need for public health strategies to help improve dietary quality and reduce chronic disease risk is becoming more crucial ${ }^{(3)}$.

Public health recommendations advise limiting red meat consumption to $\leq 500 \mathrm{~g} /$ week $^{(4)}$. However, there is conflicting evidence regarding the type of red meat consumed. A number of reviews have highlighted the importance of discriminating processed from unprocessed red meat when examining the effect on health outcomes ${ }^{(2,5)}$. This suggests that preservation and processing, coupled with the confounding effects of diet and lifestyle factors, need to be considered in disease causality ${ }^{(6)}$. A meta-analysis by Micha et al. identified an association between a $50 \mathrm{~g} / \mathrm{d}$ serving of processed meat and CVD risk (relative risk 1.42; $95 \%$ CI 1.07, 1.89, $P=0.04$ ); however, no association was observed with a $100 \mathrm{~g}$ serving of unprocessed red meat (relative risk 1.00; $95 \%$ CI $0.81,1.23, P=0.36)^{(2)}$. Moreover, processed meat consumption has also been associated with a greater incidence of T2D (hazard ratio 1.32; $95 \%$ CI 1.25, 1.40, $P<0.001$ ) than unprocessed red meat consumption (hazard ratio $1 \cdot 12$; $95 \%$ CI $1 \cdot 08,1 \cdot 16, P<0 \cdot 001)^{(5)}$.

However, because of their observational nature it is difficult to determine causality. A recent meta-analysis by O'Connor et al., which included randomised controlled trials only, failed to find a causal relationship between daily total red meat intakes of $\geq 0.5$ servings/d ( $\geq 35 \mathrm{~g} / \mathrm{d})$ and markers of CVD, cholesterol levels, TAG and blood pressure $(P>0.05)^{(7)}$. Similarly, a meta-analysis by Fretts et al. reported that the association of red meat with markers of T2D was attenuated after controlling for $\mathrm{BMI}^{(8)}$.

The above analyses were based on meat consumption; however, when reviewing the impact of food types on metabolic disease risk, it is important to consider the overall dietary pattern. Low red meat consumption $(\leq 0.5$ servings $/ d)$ is typical of a Mediterranean dietary pattern, which has been associated with lesser risk for CVD and $\mathrm{T} 2 \mathrm{D}^{(9,10)}$; however, randomised controlled trials have shown that Mediterranean diet patterns with $\geq 0 \cdot 5$

Abbreviations: \%TE, percentage of total energy; T2D, type 2 diabetes.

* Corresponding author: B. A. McNulty, fax +353 1716 1147, email breige.mcnulty@ucd.ie 
servings/d of red meat had a similar effect on CVD risk factors ${ }^{(11)}$. In contrast, higher red meat consumption is typically associated with a Western dietary pattern, which has been positively associated with increased risk of disease ${ }^{(9,10,12)}$.

The aims of this analysis were to identify dietary patterns in a nationally representative cross-sectional cohort, to characterise the contribution of processed red meat to overall diet and, moreover, to investigate the association of biomarkers of CVD and T2D with nutrient intakes and plasma fatty acid levels within identified dietary patterns.

\section{Methods \\ Study population}

The National Adult Nutrition Survey (NANS) is a cross-sectional food consumption survey of a demographically representative sample comprising 1500 free-living men $(n$ 740) and women ( $n$ 760), aged 18-90 years, across the Republic of Ireland between 2008 and $2010^{(13)}$. Individuals who failed to provide a blood sample ( $n$ 364) were excluded from the current analysis, as were under-reporters ( $n$ 351) - those participants who presented an energy intake:BMR $\leq 1 \cdot 10^{(14)}$. The final sample size was 786 (men: 399; women: 387). There were no differences in sex, age, current smoking status and supplement use between those included in the analysis and those excluded. However, there was a greater percentage of non-manual/skilled manual workers in the excluded population, who had a higher BMI than individuals in the current cohort $(P<0 \cdot 05)$. A detailed description of the NANS recruitment and methodology is reported elsewhere ${ }^{(13)}$. However, a concise overview of the data collection and laboratory techniques relevant to this analysis is outlined below. Ethical approval was granted by the University College Cork Clinical Research Ethics Committee of the Cork Teaching Hospitals and the Human Ethics Research Committee of University College Dublin (ECM 3 (p), 4 September 2008). Written consent was obtained from each participant, in accordance with the Declaration of Helsinki.

\section{Dietary assessment and analysis}

Food and beverage intakes were assessed over 4 consecutive days, including at least 1 weekend day, using a semi-weighed food diary. Brand names, recipes and cooking methods were also recorded. The food-composition database was updated to include recipes, nutritional supplements, commonly consumed generic Irish foods and new foods on the market. The database comprised 133068 rows of data and 2552 food codes were assigned to all food, beverages and nutritional supplements consumed. Food and nutrient intakes were analysed using the Weighed Intake Software Program (WISP@ version 3.0; Tinuviel Software) ${ }^{(13)}$. A total of 2048 food codes were aggregated into twenty-nine food groups, representative of the overall diet ${ }^{(15)}$. In all, 502 food codes contained meat and were characterised into four groups: unprocessed red, processed red, unprocessed white and processed white meat. Red meat included beef, lamb and pork, whereas poultry was classified as white meat. Processed meat had undergone salting, curing, fermentation, smoking, flavour enhancement or other preservation processes, examples of which included ham and sausages ${ }^{(16)}$. To calculate mean daily processed red meat intakes, each food code was updated for grams of meat per $100 \mathrm{~g}$ of product, using the online McCance and Widdowson Composition of Foods integrated data set and manufacturer's information ${ }^{(17)}$. Information on sociodemographic characteristics, health and lifestyle habits and anthropometric measurements were obtained ${ }^{(13)}$. Alternate Healthy Eating Index (AHEI) scores were assigned based on the criteria given by McCullough et $a l .{ }^{(18)}$ with a higher overall score representing a healthier diet pattern.

\section{Biochemistry measurements}

A clinical bioanalyser (RX Daytona; Randox Laboratories) was used to measure levels of glucose, TAG and total and HDL-cholesterol in serum samples ${ }^{(19)}$. LDL-cholesterol levels were calculated as (Total cholesterol/HDL-cholesterol) - (TAG/2.2). Insulin, leptin and TNF $\alpha$ levels were measured using a biochip array system (Evidence Investigator; Randox Laboratories). Adiponectin levels were measured using ELISA (ALPCO Diagnostics kit; ALPCO) and homocysteine levels using a flourescence polarisation immunoassay. A detailed description of the lipid extraction methodology and fatty acid analysis has been outlined elsewhere ${ }^{(20)}$. The National Cholesterol Education Programme's Adult Treatment Panel III criterion was applied to evaluate risk for the $\operatorname{MetS}^{(21)}$.

\section{Statistical analysis}

Data were analysed using SPSS $^{\circledR}$ for Windows ${ }^{\text {TM }}$ statistical software package version 20.0 (SPSS Inc.). Descriptive statistics for continuous variables are presented as mean values and standard deviations, whereas categorical variables are reported as percentages.

The thirty-three food groups were converted to percentages of total daily energy (\%TE) intakes, to derive dietary patterns proportional to energy intakes, and standardised as $z$ scores. Preliminary two-step cluster analysis was applied to determine the optimal number of dietary clusters in the cohort. The first step involves the formation of preclusters, based on the distance criterion, whereas the second step applies the standard hierarchical clustering algorithm to these preclusters. This analysis identified four dominant dietary patterns. $k$-Means subsequently characterised these patterns by separating participants into non-overlapping groups derived from Euclidean distance. To validate the dietary patterns, the population was randomly divided into two parts and the analysis was repeated. In all, 69\% of individuals were re-classified in the cluster analysis validation.

Sociodemographic characteristics were analysed using the $\chi^{2}$ statistic for categorical variables and one-way ANOVA with Bonferroni post boc tests for continuous variables. Differences in nutrient intakes, dietary quality, anthropometric measurements and biochemical data were assessed using an adjusted general linear model. Bonferoni correction was applied by multiplying each $P$ value by the number of traits in each table. $P \leq 0.05$ were considered significant and those that exceeded $1 \cdot 0$ were marked down to $1 \cdot 000$.

\section{Results}

Total red meat intake was $134 \mathrm{~g} / \mathrm{d}$ (male) and $89 \mathrm{~g} / \mathrm{d}$ (female), whereas processed red meat intakes were $52 \mathrm{~g} / \mathrm{d}$ (male) and 
$29 \mathrm{~g} / \mathrm{d}$ (female). Overall, four dietary patterns were identified (Table 1). Pattern 1 presented higher energy contributions from wholemeal breads, vegetables, fruit, fish and yogurts $(P<0 \cdot 001)$. The greatest contributors to energy in Pattern 2 were chips, processed potatoes, rice, pasta, fruit juices, smoothies and cheeses $(P<0 \cdot 001)$. Pattern 3 had higher contributions from alcoholic beverages, unprocessed red meat, ready-to-eat breakfast cereals, savouries and confectionery $(P<0.05)$. White bread, processed red meat, butters, whole milk and potatoes were the greatest contributors towards energy intakes in Pattern $4(P<0.001)$. Mean daily processed red meat intakes were lowest in Pattern 1 (1.3\%TE) and greatest in Pattern $4(2.4 \%$ TE $)(P<0.001)$. There was a significantly greater contribution of processed red meat to Pattern 4, in comparison with all other patterns (online Supplementary Table S1).

Participants in Pattern 1 were predominantly older professional women, with better dietary quality and greater supplement usage $(P<0 \cdot 001)$. Pattern 2 was seen in younger female participants, who were of a lower social class and were more likely to smoke $(P<0 \cdot 001)$. Pattern 3 was observed in younger participants, with a slightly higher proportion of male participants than female, with the greatest energy intakes $(P<0 \cdot 001)$. Participants in Pattern 4 were typically older men and women,

(Mean values and standard deviations) who presented the poorest AHEI scores $(P<0 \cdot 05)$. Participants in Patterns 3 and 4 were typically non-manual or skilled manual workers (Table 2).

Mean daily intakes, including contributions from supplements, are presented in Tables 3 and Table 4. Carbohydrate (\%TE), sugar (\%TE) and fibre $(\mathrm{g} / 10 \mathrm{MJ})$ intakes were significantly greater in Pattern $1(P<0.001)$. Total fat (\%TE) intakes were greatest in Patterns 2 and 4, with Pattern 4 presenting significantly greater SFA (\%TE) intakes than the other three patterns. MUFA (\%TE), PUFA fat (\%TE) and $\alpha$-linolenic acid (\%TE) intakes were greatest in Pattern $2(P<0.001)$, with no significant differences in EPA (\%TE) and DHA (\%TE) levels between patterns. $\mathrm{Na}(\mathrm{mg} / 10 \mathrm{MJ})$ intakes were significantly lower, whereas intakes of other micronutrients were significantly greater in Pattern 1 (Table 3). In comparison with the other dietary patterns, plasma EPA (C20:5n-3) and DHA (C22:6n-3) levels were higher in Pattern $1(P<0 \cdot 001)$. No significant differences were observed in a suite of markers of CVD and T2D (Table 4).

\section{Discussion}

Overall, four dietary patterns were derived, which were distinguishable by both processed red meat consumption and other dominant food groups. The pattern with greater processed red

Table 1. Percentage of energy contribution of food groups across four dietary patterns in Irish adults

\begin{tabular}{|c|c|c|c|c|c|c|c|c|c|}
\hline \multirow[b]{2}{*}{ Food groups } & \multicolumn{2}{|c|}{ Pattern 1 (n 131) } & \multicolumn{2}{|c|}{ Pattern 2 (n 70) } & \multicolumn{2}{|c|}{ Pattern 3 (n 405) } & \multicolumn{2}{|c|}{ Pattern 4 ( $n$ 180) } & \multirow[b]{2}{*}{$P^{\star}$} \\
\hline & Mean & SD & Mean & $\mathrm{SD}$ & Mean & SD & Mean & SD & \\
\hline Processed red meat & $2 \cdot 65^{a}$ & 3.55 & $4 \cdot 21^{a, b}$ & $3 \cdot 94$ & $4.46^{b}$ & $4 \cdot 20$ & $7 \cdot 86^{\mathrm{c}}$ & $7 \cdot 13$ & $<0.001$ \\
\hline Unprocessed red meat & $5 \cdot 34^{a}$ & 4.67 & $4.64^{\mathrm{a}}$ & $4 \cdot 30$ & $7 \cdot 12^{\mathrm{b}}$ & 5.93 & $7 \cdot 08^{b}$ & $5 \cdot 31$ & $<0.001$ \\
\hline Processed white meat & 0.91 & 2.84 & 1.82 & $2 \cdot 22$ & 1.86 & $3 \cdot 19$ & $1 \cdot 16$ & 2.52 & 0.066 \\
\hline Unprocessed white meat & 3.07 & 3.57 & $4 \cdot 23$ & $4 \cdot 78$ & 3.57 & $4 \cdot 14$ & $2 \cdot 58$ & $3 \cdot 10$ & 0.198 \\
\hline Alcoholic beverages & $3.56^{a}$ & 4.91 & $7.94^{\mathrm{b}}$ & $8 \cdot 72$ & $8 \cdot 16^{b}$ & 8.88 & $3 \cdot 54^{a}$ & $5 \cdot 18$ & $<0.001$ \\
\hline Biscuits, cakes and pastries & $6 \cdot 76^{a}$ & 5.57 & $3.95^{\mathrm{b}}$ & 4.67 & $6 \cdot 74^{\mathrm{a}}$ & $6 \cdot 46$ & $5 \cdot 61^{a, b}$ & 5.55 & 0.033 \\
\hline Butters, fat spreads and cooking fats & $2 \cdot 40^{\mathrm{a}}$ & $2 \cdot 87$ & $2 \cdot 38^{a}$ & $2 \cdot 25$ & $2 \cdot 54^{\mathrm{a}}$ & 2.54 & $6 \cdot 83^{\mathrm{b}}$ & 6.79 & $<0.001$ \\
\hline Cheeses & $1.98^{\mathrm{a}, \mathrm{c}}$ & $2 \cdot 20$ & $3.33^{\mathrm{b}}$ & $2 \cdot 87$ & $2 \cdot 45^{\mathrm{a}, \mathrm{b}}$ & 2.91 & $1.82^{\mathrm{C}}$ & $2 \cdot 37$ & $<0.001$ \\
\hline Chips and processed potatoes & $2 \cdot 71^{\mathrm{a}}$ & 3.06 & $5.09^{b}$ & $5 \cdot 22$ & $4 \cdot 84^{b}$ & 4.82 & $4 \cdot 71^{b}$ & $5 \cdot 37$ & $<0.001$ \\
\hline Confectionery & $2 \cdot 56^{\mathrm{a}}$ & $3 \cdot 26$ & $3.51^{a, b}$ & $4 \cdot 19$ & $3.61^{b}$ & $4 \cdot 30$ & $2 \cdot 20^{\mathrm{a}}$ & 2.92 & 0.033 \\
\hline Creams, ice creams and desserts & $3 \cdot 15^{\mathrm{a}}$ & $4 \cdot 36$ & $2 \cdot 79^{\mathrm{a}, \mathrm{b}}$ & $4 \cdot 21$ & $1.99^{b}$ & 2.96 & $1.69^{b}$ & $2 \cdot 71$ & $<0.001$ \\
\hline Egg and egg dishes & 1.92 & 2.59 & 1.60 & 2.65 & 1.35 & 1.92 & $1 \cdot 26$ & 1.60 & 0.759 \\
\hline Fish, fish dishes and products & $4 \cdot 25^{a}$ & 4.55 & $1.84^{b}$ & $2 \cdot 70$ & $1.94^{\mathrm{b}}$ & 2.86 & $1.68^{b}$ & 2.59 & $<0.001$ \\
\hline Fruit & $6 \cdot 42^{\mathrm{a}}$ & $4 \cdot 51$ & $1.73^{\mathrm{b}}$ & $2 \cdot 42$ & $2.06^{\mathrm{b}}$ & 2.04 & $1.91^{b}$ & 2.52 & $<0.001$ \\
\hline Fruit juices and smoothies & $1 \cdot 40^{\mathrm{a}, \mathrm{b}}$ & 2.42 & $1.63^{\mathrm{a}, \mathrm{c}}$ & 1.88 & $0.96^{\mathrm{b}}$ & 1.60 & $0.64^{c}$ & 1.45 & $<0.001$ \\
\hline High-energy beverages & 0.85 & $2 \cdot 13$ & 1.95 & $3 \cdot 19$ & 1.85 & 2.96 & 1.51 & $2 \cdot 81$ & 0.198 \\
\hline Low-energy beverages & $0.31^{a}$ & 0.82 & $0 \cdot 11^{\mathrm{a}, \mathrm{b}}$ & 0.46 & $0 \cdot 10^{\mathrm{b}}$ & 0.34 & $0 \cdot 15^{\mathrm{a}, \mathrm{b}}$ & 0.59 & 0.033 \\
\hline Low-fat and skimmed milk & $2 \cdot 25$ & $2 \cdot 73$ & 1.33 & $1 \cdot 78$ & $2 \cdot 31$ & 3.54 & 1.86 & 3.46 & 0.264 \\
\hline Low-fat spreads and oils & $1 \cdot 20^{\mathrm{a}, \mathrm{c}}$ & 1.91 & $0.47^{\mathrm{a}, \mathrm{b}}$ & $1 \cdot 15$ & $0.69^{b}$ & 1.53 & $1.33^{c}$ & $2 \cdot 70$ & $<0.001$ \\
\hline Other breakfast cereals & 2.23 & 3.74 & 1.53 & $3 \cdot 15$ & $1 \cdot 15$ & $2 \cdot 83$ & 1.92 & $4 \cdot 33$ & 0.165 \\
\hline Other milk products and milk-based beverages & 0.62 & 1.57 & 0.46 & $1 \cdot 13$ & 0.42 & 1.45 & 0.23 & $1 \cdot 22$ & 1.000 \\
\hline Potatoes & $2.97^{\mathrm{a}}$ & 2.65 & $1.61^{\mathrm{b}}$ & $2 \cdot 15$ & $2 \cdot 21^{\mathrm{b}}$ & $2 \cdot 14$ & $4 \cdot 13^{c}$ & 3.68 & $<0.001$ \\
\hline Rice, pasta, flours and starches & $2 \cdot 80^{\mathrm{a}, \mathrm{b}}$ & $4 \cdot 11$ & $3 \cdot 24^{\mathrm{a}}$ & $3 \cdot 88$ & $2 \cdot 11^{\mathrm{b}}$ & $2 \cdot 84$ & $1.03^{\mathrm{C}}$ & 1.97 & $<0.001$ \\
\hline Ready-to-eat breakfast cereals & $3.67^{\mathrm{a}}$ & 4.59 & $2 \cdot 40^{\mathrm{a}}$ & 2.85 & $5 \cdot 07^{b}$ & 5.05 & $2.64^{a}$ & 3.41 & $<0.001$ \\
\hline Savouries & $1.78^{a}$ & $3 \cdot 16$ & $3.02^{a, b}$ & 4.56 & $4 \cdot 31^{b}$ & $6 \cdot 16$ & $1.95^{\mathrm{a}}$ & $4 \cdot 15$ & $<0.001$ \\
\hline Savoury snacks & $3 \cdot 78^{a}$ & $5 \cdot 74$ & $2 \cdot 02^{\mathrm{b}, \mathrm{c}}$ & $2 \cdot 22$ & $2 \cdot 67^{b}$ & 3.83 & $1.41^{\mathrm{c}}$ & 2.56 & $<0.001$ \\
\hline Soups, sauces and condiments & $2 \cdot 55^{a}$ & $2 \cdot 42$ & $1.91^{\mathrm{b}}$ & 1.59 & $1.96^{\mathrm{C}}$ & 1.60 & $1.73^{\mathrm{c}}$ & 1.91 & $<0.001$ \\
\hline Sugars, syrups, preserves and sweeteners & $1.57^{\mathrm{a}}$ & $2 \cdot 25$ & $1 \cdot 24^{\mathrm{a}}$ & 1.67 & $1.37^{\mathrm{a}}$ & 1.89 & $3 \cdot 11^{\mathrm{b}}$ & 4.04 & $<0.001$ \\
\hline Vegetables and vegetable dishes & $7 \cdot 06^{\mathrm{a}}$ & $4 \cdot 71$ & $3.25^{\mathrm{b}}$ & 2.67 & $2.55^{\mathrm{b}}$ & $1 \cdot 78$ & $2 \cdot 51^{b}$ & 2.03 & $<0.001$ \\
\hline White bread, rolls, scones and croissants & $4 \cdot 75^{\mathrm{a}}$ & 4.35 & $7 \cdot 83^{\mathrm{b}}$ & $5 \cdot 84$ & $6 \cdot 00^{a, b}$ & 4.48 & $13 \cdot 78^{c}$ & $7 \cdot 81$ & $<0.001$ \\
\hline Wholemeal, brown bread and rolls & $7 \cdot 98^{\mathrm{a}}$ & $6 \cdot 06$ & $5 \cdot 03^{\mathrm{b}, \mathrm{c}}$ & $4 \cdot 86$ & $6 \cdot 46^{\mathrm{a}, \mathrm{b}}$ & $6 \cdot 12$ & $4 \cdot 22^{\mathrm{C}}$ & $5 \cdot 47$ & $<0.001$ \\
\hline Whole milk & $1.52^{\mathrm{a}}$ & 3.01 & $2 \cdot 47^{\mathrm{a}, \mathrm{b}}$ & $3 \cdot 41$ & $3.42^{\mathrm{b}}$ & $4 \cdot 62$ & $4.65^{c}$ & $6 \cdot 12$ & $<0.001$ \\
\hline Yogurts & $2.04^{a}$ & 2.63 & $1.03^{b}$ & 1.83 & $1 \cdot 23^{b}$ & 2.00 & $0.89^{b}$ & 1.76 & $<0.001$ \\
\hline
\end{tabular}

a,b,c Mean values with unlike superscript letters are significantly different between groups $(P<0.05)$.

* Differences across dietary patterns were assessed using one-way ANOVA. Bonferroni correction was applied by multiplying the $P$ values by the number of traits in the table. $P$ values that exceeded 1.0 have been marked down to 1.000 . 
Table 2. Participant characteristics across the four dietary patterns

(Mean values and standard deviations)

\begin{tabular}{|c|c|c|c|c|c|c|c|c|c|}
\hline & \multicolumn{2}{|c|}{ Pattern 1 (n 131) } & \multicolumn{2}{|c|}{ Pattern 2 (n 70) } & \multicolumn{2}{|c|}{ Pattern 3 (n 405) } & \multicolumn{2}{|c|}{ Pattern 4 (n 180) } & \multirow[b]{2}{*}{$P \S$} \\
\hline & Mean & SD & Mean & SD & Mean & SD & Mean & SD & \\
\hline Processed red meat $(\mathrm{g} / \mathrm{d})^{\star}$ & $25 \cdot 8^{a}$ & $22 \cdot 9$ & $39.2^{b}$ & $35 \cdot 0$ & $34 \cdot 3^{\mathrm{a}, \mathrm{b}}$ & $22 \cdot 7$ & $57 \cdot 1^{\mathrm{c}}$ & $46 \cdot 6$ & $<0.001$ \\
\hline Unprocessed red meat $(\mathrm{g} / \mathrm{d})^{*}$ & $66 \cdot 9^{\mathrm{a}}$ & $43 \cdot 0$ & $95 \cdot 9^{b}$ & 63.5 & $75 \cdot 7^{\mathrm{a}, \mathrm{b}}$ & $56 \cdot 9$ & $86 \cdot 2^{a, b}$ & 57.9 & $<0.001$ \\
\hline Processed white meat $(\mathrm{g} / \mathrm{d})^{\star}$ & $26 \cdot 7$ & $27 \cdot 8$ & $32 \cdot 8$ & $29 \cdot 4$ & $22 \cdot 7$ & $23 \cdot 5$ & $29 \cdot 2$ & $23 \cdot 4$ & 1.000 \\
\hline Unprocessed white meat $(\mathrm{g} / \mathrm{d})^{\star}$ & $69 \cdot 6$ & $50 \cdot 3$ & $76 \cdot 1$ & $61 \cdot 8$ & $80 \cdot 0$ & $60 \cdot 2$ & $62 \cdot 4$ & $40 \cdot 8$ & 1.000 \\
\hline Sex (male/female, \%)† & \multicolumn{2}{|c|}{$34 / 66$} & \multicolumn{2}{|c|}{$36 / 64$} & \multicolumn{2}{|c|}{$56 / 44$} & \multicolumn{2}{|c|}{$57 / 43$} & $<0.001$ \\
\hline Age (years) ${ }^{*}$ & $48 \cdot 4^{a}$ & $15 \cdot 7$ & $33 \cdot 4^{\mathrm{b}}$ & 13.0 & $38 \cdot 9^{c}$ & $15 \cdot 9$ & $48 \cdot 6^{a}$ & $17 \cdot 2$ & $<0.001$ \\
\hline Social class $(\%) \dagger$ & \multirow{2}{*}{\multicolumn{2}{|c|}{65.4}} & & & & & & & \\
\hline Professional & & & \multicolumn{2}{|c|}{$50 \cdot 7$} & \multicolumn{2}{|c|}{44.4} & \multicolumn{2}{|c|}{$41 \cdot 7$} & $<0.001$ \\
\hline Non-manual & \multicolumn{2}{|c|}{15} & \multicolumn{2}{|c|}{$16 \cdot 4$} & \multicolumn{2}{|c|}{$16 \cdot 2$} & \multicolumn{2}{|c|}{$14 \cdot 3$} & \\
\hline Skilled manual & \multicolumn{2}{|c|}{$10 \cdot 2$} & \multicolumn{2}{|c|}{$3 \cdot 0$} & \multicolumn{2}{|c|}{$13 \cdot 4$} & \multicolumn{2}{|c|}{$21 \cdot 7$} & \\
\hline Unskilled & \multicolumn{2}{|c|}{$9 \cdot 4$} & \multicolumn{2}{|c|}{$29 \cdot 9$} & \multicolumn{2}{|c|}{$26 \cdot 0$} & \multicolumn{2}{|c|}{$22 \cdot 3$} & \\
\hline Smoker (\%)† & \multicolumn{2}{|c|}{13} & \multirow{2}{*}{\multicolumn{2}{|c|}{$\begin{array}{l}32.9 \\
31.4\end{array}$}} & \multicolumn{2}{|c|}{$16 \cdot 6$} & \multicolumn{2}{|c|}{$28 \cdot 9$} & $<0.001$ \\
\hline Supplement user (\%)† & \multicolumn{2}{|c|}{$48 \cdot 9$} & & & & & & & $<0.001$ \\
\hline Physical activity (h/week)* & $84 \cdot 3$ & $58 \cdot 1$ & $114 \cdot 3$ & $104 \cdot 8$ & $103 \cdot 8$ & $84 \cdot 2$ & $90 \cdot 0$ & $85 \cdot 7$ & 0.400 \\
\hline Energy $(\mathrm{kJ} / \mathrm{d})$ & $8636^{a}$ & 2381 & $9728^{\mathrm{b}, \mathrm{c}}$ & 2356 & $10134^{\mathrm{b}}$ & 2636 & $9360^{\mathrm{a}, \mathrm{c}}$ & 2360 & $<0.001$ \\
\hline Energy (kcal/d)* & $2064^{a}$ & 569 & $2325^{\mathrm{b}, \mathrm{c}}$ & 563 & $2422^{b}$ & 630 & $2237^{\mathrm{a}, \mathrm{c}}$ & 564 & $<0.001$ \\
\hline BMI $\left(\mathrm{kg} / \mathrm{m}^{2}\right) \ddagger$ & 25.4 & 3.9 & 25.7 & 4.5 & $26 \cdot 0$ & 4.0 & $26 \cdot 8$ & 4.4 & 1.000 \\
\hline Body fat (\%)‡ & $29 \cdot 3$ & 8.0 & $28 \cdot 1$ & $9 \cdot 6$ & $26 \cdot 6$ & $9 \cdot 3$ & 28 & $8 \cdot 5$ & 1.000 \\
\hline Muscle mass $(\mathrm{kg}) \ddagger$ & $47 \cdot 6$ & $10 \cdot 2$ & $50 \cdot 9$ & $10 \cdot 7$ & 53 & $11 \cdot 1$ & $52 \cdot 1$ & $10 \cdot 1$ & 1.000 \\
\hline Waist:hip ratio $(\mathrm{cm}) \ddagger$ & 0.86 & 0.08 & 0.83 & 0.07 & 0.87 & 0.08 & 0.9 & 0.09 & 0.920 \\
\hline Systolic BP (mmHg) & $121 \cdot 8$ & $17 \cdot 7$ & $118 \cdot 1$ & $15 \cdot 5$ & $124 \cdot 3$ & $17 \cdot 1$ & $126 \cdot 8$ & $20 \cdot 1$ & 1.000 \\
\hline Diastolic BP (mmHg) $\ddagger$ & 77.6 & $11 \cdot 2$ & 75.9 & $10 \cdot 3$ & 77.6 & 11 & 79 & 11.5 & 1.000 \\
\hline Metabolic syndrome (\%)† & & & & & & & & & 0.240 \\
\hline Alternate Healthy Eating Index $\ddagger$ & $34 \cdot 4^{\mathrm{a}}$ & $9 \cdot 9$ & $25 \cdot 7^{b}$ & $7 \cdot 4$ & $24 \cdot 9^{b}$ & $8 \cdot 5$ & $21 \cdot 2^{c}$ & $7 \cdot 7$ & $<0.001$ \\
\hline
\end{tabular}

BP, blood pressure.

a,b,c Mean values with unlike superscript letters are significantly different between groups $(P<0.05)$

* Differences across meat consumption and demographics were assessed by one-way ANOVA.

$\dagger$ Differences across sex, social class, smoking and supplement use were assessed using the Pearson $x^{2}$ Statistic.

‡ Differences across anthropometric measurements and dietary quality were assessed using a general linear model adjusted for age, sex, energy, social class, smoking status and supplement usage.

$\S$ Bonferroni correction was applied by multiplying the $P$ values by the number of traits in the table. $P$ values that exceeded 1.0 have been marked down to 1.000 .

Table 3. Nutrient composition across four dietary patterns

(Mean values and standard deviations)

\begin{tabular}{|c|c|c|c|c|c|c|c|c|c|}
\hline & \multicolumn{2}{|c|}{ Pattern 1 ( $n$ 131) } & \multicolumn{2}{|c|}{ Pattern 2 (n 70) } & \multicolumn{2}{|c|}{ Pattern 3 (n 405) } & \multicolumn{2}{|c|}{ Pattern 4 (n 180) } & \multirow[b]{2}{*}{$P^{\star}$} \\
\hline & Mean & SD & Mean & SD & Mean & SD & Mean & SD & \\
\hline Carbohydrate (\%TE) & $44 \cdot 6^{a}$ & $6 \cdot 4$ & $39.6^{\mathrm{b}}$ & $5 \cdot 7$ & $42 \cdot 3^{a}$ & $6 \cdot 8$ & $43.0^{a}$ & $7 \cdot 0$ & $<0.001$ \\
\hline Sugars (\%TE) & $19 \cdot 9^{a}$ & $5 \cdot 6$ & $15 \cdot 7^{\mathrm{b}}$ & $4 \cdot 4$ & $16 \cdot 9^{b}$ & $5 \cdot 2$ & $16 \cdot 4^{b}$ & $6 \cdot 2$ & $<0.001$ \\
\hline Protein (\%TE) & $16 \cdot 8$ & 3.4 & $15 \cdot 2$ & $3 \cdot 0$ & $16 \cdot 2$ & $3 \cdot 2$ & $16 \cdot 0$ & $3 \cdot 1$ & 1.000 \\
\hline Total fat (\%TE) & $34 \cdot 2^{a, b}$ & $6 \cdot 2$ & $36 \cdot 0^{b, c}$ & $6 \cdot 7$ & $33 \cdot 1^{a}$ & $5 \cdot 7$ & $36 \cdot 0^{\mathrm{C}}$ & $6 \cdot 1$ & $<0.001$ \\
\hline SFA (\%TE) & $12 \cdot 6^{a}$ & 3.3 & $12 \cdot 7^{\mathrm{a}}$ & $3 \cdot 2$ & $13 \cdot 1^{\mathrm{a}}$ & $3 \cdot 1$ & $14 \cdot 7^{\mathrm{b}}$ & 3.7 & $<0.001$ \\
\hline MUFA (\%TE) & $12 \cdot 2^{\mathrm{a}, \mathrm{c}}$ & 2.5 & $13 \cdot 9^{a}$ & $3 \cdot 2$ & $12 \cdot 1^{\mathrm{b}}$ & $2 \cdot 3$ & $13 \cdot 1^{\mathrm{b}, \mathrm{c}}$ & $2 \cdot 7$ & $<0.001$ \\
\hline PUFA (\%TE) & $7 \cdot 2^{a}$ & $2 \cdot 7$ & $7 \cdot 2^{a}$ & 2.4 & $5 \cdot 8^{b}$ & $2 \cdot 0$ & $5 \cdot 9^{b}$ & $2 \cdot 3$ & $<0.001$ \\
\hline ALA (\%TE) & $0 \cdot 7^{\mathrm{a}}$ & 0.4 & $0.8^{a}$ & 0.3 & $0.5^{\mathrm{b}}$ & 0.2 & $0.6^{a}$ & 0.6 & $<0.001$ \\
\hline EPA (\%TE) & 0.12 & 0.67 & 0.01 & 0.04 & 0.02 & 0.17 & 0.03 & 0.23 & 1.000 \\
\hline $\mathrm{DHA}(\% \mathrm{TE})$ & 0.17 & 0.66 & 0.01 & 0.04 & 0.02 & 0.16 & 0.03 & 0.23 & 1.000 \\
\hline Fibre (g/10 MJ) & $30 \cdot 6^{a}$ & $8 \cdot 4$ & $20 \cdot 0^{b, c}$ & $6 \cdot 2$ & $21 \cdot 4^{\mathrm{b}}$ & $6 \cdot 7$ & $19 \cdot 9^{c}$ & $6 \cdot 6$ & $<0.001$ \\
\hline Vitamin A ( $\mu \mathrm{g} / 10 \mathrm{MJ})$ & $1830 \cdot 5^{a}$ & $1146 \cdot 2$ & $1095 \cdot 6^{\mathrm{a}, \mathrm{b}}$ & $766 \cdot 1$ & $1179 \cdot 5^{b}$ & $988 \cdot 2$ & $1208 \cdot 2^{b}$ & $809 \cdot 5$ & $<0.001$ \\
\hline Vitamin $\mathrm{B}_{6}(\mathrm{mg} / 10 \mathrm{MJ})$ & 6.6 & $13 \cdot 1$ & 3.7 & 4.8 & 4.6 & $9 \cdot 1$ & 3.9 & $7 \cdot 3$ & 1.000 \\
\hline Vitamin $B_{12}(\mu \mathrm{g} / 10 \mathrm{MJ})$ & $21 \cdot 3$ & 113.4 & 5.4 & 4.5 & $6 \cdot 6$ & $10 \cdot 0$ & $6 \cdot 1$ & $7 \cdot 8$ & 1.000 \\
\hline Biotin $(\mu \mathrm{g} / 10 \mathrm{MJ})$ & $91 \cdot 2$ & $207 \cdot 0$ & $45 \cdot 0$ & $28 \cdot 2$ & $54 \cdot 7$ & $43 \cdot 1$ & $47 \cdot 1$ & $30 \cdot 6$ & 0.572 \\
\hline Riboflavin (mg/10 MJ) & 5.4 & $12 \cdot 7$ & 3.1 & 5.9 & 3.9 & 9.5 & 3.0 & $9 \cdot 8$ & 1.000 \\
\hline Vitamin C (mg/10 MJ) & $269 \cdot 1^{a}$ & $446 \cdot 2$ & $134 \cdot 8^{\mathrm{b}}$ & $110 \cdot 6$ & $125 \cdot 9^{\mathrm{b}}$ & $180 \cdot 4$ & $100 \cdot 0^{\mathrm{b}}$ & $122 \cdot 9$ & $<0.001$ \\
\hline Vitamin $D(\mu \mathrm{g} / 10 \mathrm{MJ})$ & $9 \cdot 7^{\mathrm{a}}$ & $14 \cdot \overline{6}$ & $3.4^{\mathrm{b}}$ & $4 \cdot 1$ & $4 \cdot 6^{\mathrm{b}}$ & $5 \cdot 0$ & $5 \cdot 1^{\mathrm{b}}$ & 5.7 & $<0.001$ \\
\hline $\mathrm{Ca}(\mathrm{mg} / 10 \mathrm{MJ})$ & 1191.0 & 534.3 & $1008 \cdot 7$ & $394 \cdot 8$ & 1099.5 & 390.5 & $1112 \cdot 3$ & $431 \cdot 7$ & 1.000 \\
\hline $\mathrm{Cu}(\mathrm{mg} / 10 \mathrm{MJ})$ & 1.8 & $1 \cdot 1$ & $1 \cdot 2$ & 0.4 & 1.4 & 1.8 & 1.2 & 0.8 & 1.000 \\
\hline $\mathrm{Fe}(\mathrm{mg} / 10 \mathrm{MJ})$ & $17 \cdot 9$ & $10 \cdot 6$ & 14.5 & 8.5 & $16 \cdot 3$ & $17 \cdot 1$ & $16 \cdot 8$ & $20 \cdot 9$ & 1.000 \\
\hline $\mathrm{Mg}(\mathrm{mg} / 10 \mathrm{MJ})$ & $417 \cdot 5^{a}$ & $152 \cdot 2$ & $312 \cdot 7^{\mathrm{b}, \mathrm{c}}$ & $64 \cdot 2$ & $340 \cdot 2^{\mathrm{b}}$ & $79 \cdot 1$ & $307 \cdot 4^{\mathrm{c}}$ & $73 \cdot 0$ & $<0.001$ \\
\hline $\mathrm{K}(\mathrm{mg} / 10 \mathrm{MJ})$ & $4115 \cdot 3^{a}$ & 705.8 & $3419 \cdot 6^{\mathrm{b}, \mathrm{c}}$ & 564.5 & $398 \cdot 0^{\mathrm{b}}$ & 771.3 & $3389 \cdot 0^{c}$ & $747 \cdot 2$ & $<0.001$ \\
\hline $\mathrm{P}(\mathrm{mg} / 10 \mathrm{MJ})$ & $1735 \cdot 3^{a}$ & $301 \cdot 1$ & $1495 \cdot 5^{b}$ & $286 \cdot 7$ & $1599 \cdot 2^{\mathrm{a}, \mathrm{b}}$ & 277.5 & $1561 \cdot 4^{b}$ & 339.5 & $<0.001$ \\
\hline $\mathrm{Na}(\mathrm{mg} / 10 \mathrm{MJ})$ & $2787 \cdot 8^{a}$ & 638.2 & $2902 \cdot 1^{a, b}$ & $454 \cdot 8$ & $2838 \cdot 3^{a}$ & 601.5 & $3106 \cdot 9^{b}$ & 610.9 & $<0.001$ \\
\hline $\mathrm{Zn}(\mathrm{mg} / 10 \mathrm{MJ})$ & $15 \cdot 8$ & $17 \cdot 4$ & 9.6 & 2.9 & 11.6 & 4.9 & 11.7 & $7 \cdot 1$ & 0.130 \\
\hline
\end{tabular}

$\%$ TE, percentage of total energy; ALA, a-linolenic acid.

a,b,c Mean values with unlike superscript letters are significantly different between groups $(P<0.05)$

* Differences in nutrient intakes across dietary patterns were assessed using a General Linear Model adjusted for age, sex, social class, smoking status and supplement usage. Bonferroni correction was applied by multiplying the $P$ values by the number of traits in the table. $P$ values that exceeded 1.0 have been marked down to 1.000 . 
Table 4. Plasma fatty acid levels and markers of metabolic health across the four dietary patterns (Mean values and standard deviations)

\begin{tabular}{|c|c|c|c|c|c|c|c|c|c|c|}
\hline & \multicolumn{2}{|c|}{ Pattern 1 (n 100-130) } & \multicolumn{2}{|c|}{ Pattern 2 ( $n$ 57-70) } & \multicolumn{2}{|c|}{ Pattern 3 ( $n$ 291-403) } & \multicolumn{2}{|c|}{ Pattern 4 (n 130-179) } & \multirow[b]{2}{*}{$P^{*}$} & \multirow[b]{2}{*}{$P \dagger$} \\
\hline & Mean & SD & Mean & SD & Mean & SD & Mean & SD & & \\
\hline \multicolumn{11}{|l|}{$\%$ Total plasma fatty acids } \\
\hline C15:0 & 0.33 & 0.09 & 0.32 & 0.10 & 0.38 & 1.04 & 0.35 & 0.10 & 0.900 & 1.000 \\
\hline C16:0 & $22 \cdot 60$ & 2.92 & $22 \cdot 50$ & 1.64 & 23.00 & $2 \cdot 22$ & 22.91 & 1.95 & 0.146 & 1.000 \\
\hline C16:1 & $2 \cdot 23^{\mathrm{a}}$ & 0.74 & $2 \cdot 34^{a, b}$ & $6 \cdot 70$ & $2 \cdot 56^{\mathrm{b}}$ & 0.88 & $2 \cdot 62^{\mathrm{b}}$ & 0.89 & $<0.001$ & $<0.001$ \\
\hline C18: $1 n-9$ & 17.90 & $3 \cdot 21$ & $18 \cdot 50$ & 4.47 & $18 \cdot 20$ & 3.42 & $19 \cdot 03$ & $4 \cdot 15$ & 0.035 & 1.000 \\
\hline C18:3n-3 & 0.81 & 0.28 & 0.88 & 0.30 & 0.83 & 0.25 & 0.93 & 0.36 & 0.001 & 0.456 \\
\hline C18:3n-6 & 0.51 & $0 \cdot 19$ & 0.58 & 0.30 & 0.56 & 0.17 & 0.56 & 0.20 & 0.058 & 0.144 \\
\hline $\mathrm{C} 20: 1 n-9$ & 0.24 & 0.07 & 0.23 & 0.09 & 0.22 & 0.96 & 0.23 & 0.07 & 0.030 & 0.816 \\
\hline $\mathrm{C} 20: 3 n-6$ & $1.87^{\mathrm{a}}$ & 0.50 & $1.95^{\mathrm{a}, \mathrm{b}}$ & 0.43 & $2 \cdot 08^{\mathrm{b}, \mathrm{c}}$ & 0.48 & $2 \cdot 07^{\mathrm{c}}$ & 0.42 & $<0.001$ & $<0.001$ \\
\hline C20 : 4n-6 & 7.69 & 2.06 & 7.73 & 1.98 & 7.57 & 1.86 & 7.44 & 1.93 & 0.611 & 1.000 \\
\hline $\mathrm{C} 20: 5 n-3$ & $1.83^{a}$ & 1.42 & $1 \cdot 27^{\mathrm{a}, \mathrm{b}}$ & 0.61 & $1 \cdot 26^{b}$ & 0.70 & $1 \cdot 34^{\mathrm{b}}$ & 0.72 & $<0.001$ & $<0.001$ \\
\hline C22: $4 n-6$ & 0.23 & 0.08 & 0.22 & 0.08 & 0.24 & 0.09 & 0.25 & 0.10 & 0.070 & 1.000 \\
\hline $\mathrm{C} 22: 6 n-3$ & $3.03^{a}$ & $1 \cdot 20$ & $2 \cdot 30^{\mathrm{b}}$ & 0.81 & $2 \cdot 30^{\mathrm{b}}$ & 0.81 & $2 \cdot 21^{\mathrm{b}}$ & 0.84 & $<0.001$ & $<0.001$ \\
\hline $\mathrm{C} 23: 0$ & 0.60 & $4 \cdot 33$ & $0 \cdot 16$ & 0.07 & 0.18 & 0.09 & 0.17 & 0.09 & 0.123 & 1.000 \\
\hline $\mathrm{C} 24: 0$ & 0.25 & 0.15 & 0.24 & 0.10 & 0.26 & 0.14 & 0.24 & 0.10 & 0.223 & 1.000 \\
\hline \multicolumn{11}{|l|}{ Metabolic health } \\
\hline Glucose $(\mathrm{mmol} / \mathrm{l})$ & $5 \cdot 19$ & 0.88 & 5.09 & 0.73 & $5 \cdot 20$ & 0.81 & 4.58 & 1.31 & 0.004 & 1.000 \\
\hline Insulin $(\mu \mathrm{l} \mathrm{U} / \mathrm{ml})$ & 9.52 & 10.97 & $10 \cdot 30$ & 9.48 & $12 \cdot 10$ & $13 \cdot 73$ & $15 \cdot 82$ & $25 \cdot 79$ & 0.006 & 1.000 \\
\hline TAG (mmol/l) & $1 \cdot 11$ & 0.59 & 1.09 & 0.65 & 1.32 & 0.83 & 1.41 & 0.82 & 0.001 & 1.000 \\
\hline Total cholesterol $(\mathrm{mmol} / \mathrm{l})$ & $5 \cdot 01$ & 0.92 & 4.72 & 0.90 & 4.94 & 1.06 & 4.97 & 0.99 & 0.255 & 1.000 \\
\hline HDL-cholesterol(mmol/l) & $1 \cdot 70$ & 0.48 & 1.66 & 0.39 & 1.55 & 0.40 & 1.54 & 0.41 & 0.001 & 1.000 \\
\hline LDL-cholesterol (mmol/li) & $2 \cdot 78$ & $0 \cdot 78$ & $2 \cdot 57$ & 0.77 & $2 \cdot 80$ & 0.88 & $2 \cdot 80$ & 0.93 & 0.245 & 1.000 \\
\hline Adiponectin $(\mu \mathrm{g} / \mathrm{ml})$ & 7.08 & 4.51 & 6.59 & 3.01 & 5.89 & 2.93 & $5 \cdot 81$ & $2 \cdot 68$ & 0.001 & 1.000 \\
\hline Leptin (ng/ml) & 5.29 & 6.03 & $6 \cdot 14$ & 5.85 & 5.29 & $6 \cdot 85$ & $5 \cdot 10$ & 6.85 & 0.798 & 1.000 \\
\hline Homocysteine $(\mathrm{mmol} / \mathrm{l})$ & 11.5 & 3.92 & 11.9 & $2 \cdot 88$ & $12 \cdot 00$ & 3.35 & $13 \cdot 49$ & 5.01 & $<0.001$ & 0.672 \\
\hline TNFa $(p g / m l)$ & $6 \cdot 66$ & 3.05 & $6 \cdot 17$ & 1.62 & $6 \cdot 72$ & 1.96 & 7.52 & $2 \cdot 88$ & $<0.001$ & 1.000 \\
\hline
\end{tabular}

a,b,c Mean values with unlike superscript letters are significantly different between groups $(P<0.05)$

* Differences in fatty acids and markers of metabolic health across dietary patterns were assessed using a one-way ANOVA.

† Differences in fatty acids and markers of metabolic health across dietary patterns were assessed using general linear model adjusted for age, sex, energy (kJ (kcal)), social class, smoking status, supplement usage and fasting status. Bonferroni correction was applied by multiplying the $P$ values by the number of traits in the table. $P$ values that exceeded 1.0 have been marked down to 1.000

meat intakes presented a poorer AHEI score and a lower $n-3$ PUFA status compared with the pattern with significantly lower contributions from processed red meat; however, there were no significant differences in traditional biomarkers of CVD and T2D between the patterns.

Total red meat intake in the current analysis was $134 \mathrm{~g} / \mathrm{d}$ in men and $89 \mathrm{~g} / \mathrm{d}$ in women, with $46 \%$ adhering to the recommendation of $\leq 500 \mathrm{~g} /$ week $^{(4)}$. In all, $85 \%$ of the cohort consumed processed red meat; intakes of which are recommended to be limited $^{(4)}$. Men $(52 \mathrm{~g} / \mathrm{d})$ presented greater mean daily processed red meat intakes than women $(29 \mathrm{~g} / \mathrm{d})$. Irish intakes are slightly higher than intakes in the UK, similar to those in Spain, Sweden and Denmark, and lower than those in Germany ${ }^{(22-24)}$. However, it must be noted that much of the dietary intake data in the aforementioned studies were collected over a decade ago and the applied definitions differ slightly. Data exist for processed red meat intakes in the USA (male $29 \mathrm{~g} / \mathrm{d}$, female $18 \mathrm{~g} / \mathrm{d}$ ); again this is not directly comparable due to the definitions applied ${ }^{(25)}$. This lack of a stringent, global definition for processed meat is one of the major limitations when investigating processed red meat consumption as a risk factor for disease. Further research is required to ascertain whether there is greater risk associated with specific products as opposed to total processed red meat.

The current analysis included red meat that underwent smoking, salting, curing, fermentation or other processing to enhance flavour or improve preservation ${ }^{(16)}$. It is important to consider the effects of these processing techniques, and the added ingredients, including salt, which may be contributing to the observed association between processed red meat and risk for incident CVD and T2D ${ }^{(2,6)}$. In a review by Micha et al. which included twenty-seven observational studies (CVD; $n$ 10; T2D; $n$ 17) from ten countries, studies presented varying quantities of mean daily red meat intakes, with differing processed meat definitions and differing levels of confounder adjustment outlining the difficulties in reviewing this area ${ }^{(2)}$. Furthermore, high processed meat consumers were characterised by lessfavourable dietary and lifestyle factors ${ }^{(2)}$. However, recent studies are inconsistent with the aforementioned results, with no causal association observed between total red meat intakes and biomarkers of CVD using randomised controlled trials, with similar biomarker levels to the current study ${ }^{(7)}$. Further, it has been noted that the observed association between both processed and unprocessed red meat and biomarkers of T2D were attenuated following adjustment for confounding factors, particularly BMI, and multiple comparisons ${ }^{(8)}$. There were significant differences between processed red meat consumption and biomarkers of health in the current cohort; however, this was before adjustment for confounding factors and Bonferroni correction (online Supplementary Table S2).

Dietary patterns have been associated with predicting risk of disease; processed red meat is typical of the Western-style diet, 
which has been associated with an increased risk for CVD and $\mathrm{T}_{2} \mathrm{D}^{(9)}$. However, a recent study found no association between a 'meat and fish' pattern and 10-year CVD risk, whereas 'refined foods' including soft drinks and alcohol were associated with a predicted 10-year CVD risk in a Mexican cohort using factor analysis (relative risk 2.98; 95\% CI 1.46, $\left.6 \cdot 10 ; P_{\text {trend }}=0.020\right)^{(26)}$. As the majority of studies to date have focused on high processed red meat intakes, not the overall diet, and on the incidence of disease, the aim of this analysis was to characterise the contributions of red meat to dietary patterns in a European cohort. With four dominant dietary patterns derived, it was observed that Pattern 1 was similar to the Mediterranean pattern with Patterns 2 and 3 comprising components of the Western pattern ${ }^{(9)}$. Pattern 4 had a significantly greater contribution from processed red meat than the other dietary patterns. Other dominant food groups in this pattern included butters and whole milk, and lower contributions from fruit, vegetables and fish, consistent with significantly lower plasma EPA and DHA levels; however, only plasma DHA remained significant after exclusion of fish-oil supplement users ( $n$ 94). Mean EPA and DHA intake in the total population ( $n$ 786) was $120 \mathrm{mg} / \mathrm{d}$, lower than the European Food Safety Authority (EFSA) recommendation of $250 \mathrm{mg} / \mathrm{d}^{(27)}$. Pattern 1 was the sole achiever of the EPA and DHA recommendation $(342 \mathrm{~g} / \mathrm{d})$, potentially due to greater dietary intakes of fish, which was reflected in their plasma fatty acid levels.

Similar to previous studies, participants in the high processed red meat pattern were typically older and of a lower social class; $29 \%$ were current smokers with a significantly lower AHEI score and dietary fibre intake and greater SFA intakes. This is consistent with the findings of the study by Li et al. in which older participants presented greater SFA intakes ${ }^{(28)}$. However, unlike other analyses investigating processed red meat and disease risk, the current study had access to a suite of blood biomarkers of CVD and T2D, to complement the dietary intake data. Significant differences were observed between dietary patterns and biomarkers of CVD and T2D; however, this was attenuated when confounding factors were included in the model (Table 4). The possibility that red meat may be associated with increased non-traditional biomarkers should also be investigated. It is evident that further research is required to confirm the degree of association between processed red meat consumption and development of cardio-metabolic diseases, with careful consideration of the definition applied, the processing procedures and the residual confounding factors. A Mendelian randomisation approach as suggested by Rohrmann \& Linseisen may be a potential strategy ${ }^{(6)}$.

The large, nationally representative cohort, the 4-d semiweighed food diaries, and product information at brand level, coupled with metabolic biomarkers, strengthened the current analysis, whereas the inclusion of plasma fatty acid data provided a novel aspect. Dietary intakes were self-reported; thus, the removal of under-reporters eliminated potential reporting bias, and the Bonferoni correction for multiple comparisons strengthened the statistical analysis. However, the cross-sectional nature of the NANS is a limitation, as we cannot comment on the causal relationship between processed red meat and these diseases but merely state that we failed to observe an association between the dietary patterns and traditional blood biomarkers. This analysis was also limited to processed red meat contributions to dietary patterns. The application of cluster analysis to derive dietary patterns may have potentially resulted in a loss of statistical power, as it classified participants into an individual pattern, in comparison with factor analysis in which individuals receive a factor score for all derived dietary patterns. Further, the NANS was typical of a healthy cohort; findings may differ in an at-risk or diseased cohort.

In conclusion, no association was observed between high consumption of processed red meat and biomarkers of CVD and T2D in the current cohort. This finding is similar to those from the meta-analyses by O'Connor et al. and Fretts et al., who failed to find an association between red meat consumption and $\mathrm{CVD}^{(7)}$ and T2D ${ }^{(8)}$. Similar to other cohorts, high consumers of processed red meat presented a more unfavourable diet and lifestyle, which needs to be considered when investigating the association between processed red meat consumption and incidence of CVD and T2D. This analysis supports previous findings that emphasise overall dietary quality as a measure of health, rather than intakes of single foods and nutrients. Therefore, future public health recommendations should consider focusing on the total diet, based on the conflicting evidence for the role of processed red meat in disease risk. Furthermore, a global definition of processed meat should also to be developed, and modification of ingredients, similar to salt reductions, may be an effective public health strategy to improve the quality of processed red meat.

\section{Acknowledgements}

The authors are grateful for the assistance of Ricardo Segurado (Statistician at CStar, www.cstar.ie) for advice on statistical components of the study, and the NANS participants for providing detailed dietary intake data and blood samples.

This research was supported by the Irish Department of Agriculture, Food and the Marine under the National Development Plan (2007-2013) (grant no. 13/F/514) and the Health Research Board under their joint Food for Health Research Initiative (2007-2012) (grant no. FHRIUCC2).

The authors' contribution are as follows: B. A. M., A. P. N., H. M. R., L. B., A. F. and J. W. designed the research, Y. M. L., B. A. M., A. P. N. and K. F. L. conducted the research, Y. M. L. and B. A. M. analysed the data and prepared the manuscript. All authors read and approved the final manuscript.

None of the authors has any conflicts of interest to declare.

\section{Supplementary material}

For supplementary material/s referred to in this article, please visit https://doi.org/10.1017/S0007114517002008

\section{References}

1. Azadbakht L \& Esmaillzadeh A (2009) Red meat intake is associated with metabolic syndrome and the plasma C-reactive protein concentration in women. J Nutr 139, 335-339. 
2. Micha R, Michas G \& Mozaffarian D (2009) Unprocessed red and processed meats and risk of coronary artery disease and type 2 diabetes-an updated review of the evidence. Curr Atheroscler Rep 14, 515-524.

3. World Health Organization (2014) The top 10 causes of death. http://who.int/mediacentre/factsheets/fs310/en (accessed June2016).

4. World Cancer Research Fund/American Institute for Cancer Research (2007) Food, Nutrition, Physical Activity, and the Prevention of Cancer: a Global Perspective. http://www.aicr.org/ assets/docs/pdf/reports/Second_Expert_Report.pdf （accessed June 2016).

5. Pan A, Sun Q, Bernstein AM, et al. (2011) Red meat consumption and risk of type 2 diabetes: 3 cohorts of US adults and an updated meta-analysis. Am J Clin Nutr 94, 1088-1096.

6. Rohrmann S \& Linseisen J (2015) Processed meat: the real villain? Proc Nutr Soc 75, 233-241.

7. O'Connor LE, Kim JE \& Campbell WW (2017) Total red meat intake of $\geq 0.5$ servings/d does not negatively influence cardiovascular disease risk factors: a systemically searched meta-analysis of randomized controlled trials. Am J Clin Nutr 105, 57-69.

8. Fretts AM, Follis JL, Nettleton JA, et al. (2015) Consumption of meat is associated with higher fasting glucose and insulin concentrations regardless of glucose and insulin genetic risk scores: a meta-analysis of 50,345 Caucasians. Am J Clin Nutr 102, 1266-1278.

9. Heidemann C, Schulze MB, Franco OH, et al. (2008) Dietary patterns and risk of mortality from cardiovascular disease, cancer, and all causes in a prospective cohort of women. Circulation 118, 230-237.

10. van Dam RM, Rimm EB, Willett WC, et al. (2002) Dietary patterns and risk for type 2 diabetes Mellitus in U.S. men. Ann Intern Med 136, 201-209.

11. Roussell MA, Hill AM, Gaugler TL, et al. (2012) Beef in an Optimal lean diet study: effects on lipids, lipoproteins, and apolipoproteins. Am J Clin Nutr 95, 9-16.

12. Lutsey PL, Steffen LM \& Stevens J (2008) Dietary intake and the development of the metabolic syndrome: the Atherosclerosis Risk in Communities study. Circulation 117, 754-761.

13. Irish Universities Nutrition Alliance (2011) National Adult Nutrition Survey: summary report. http://www.iuna.net/wp-content/ uploads/2010/12/National-Adult-Nutrition-Survey-SummaryReport-March-2011.pdf (accessed June 2016).

14. McGowan M, Harrington K, Kiely M, et al. (2001) An evaluation of energy intakes and the ratio of energy intake to estimated basal metabolic rate (EI/BMRest) in the North/South Ireland Food Consumption Survey. Public Health Nutr 4, 1043-1050.

15. Hearty AP \& Gibney MJ (2009) Comparison of cluster and principal component analysis techniques to derive dietary patterns in Irish adults. Br J Nutr 101, 598-608.
16. Bouvard V, Loomis D, Guyton KZ, et al. (2015) Carcinogenicity of consumption of red and processed meat. Lancet Oncol 16, 1599-1600.

17. Food Standards Agency (2002) McCance and Widdowson composition of foods integrated dataset. http://www.food. gov.uk/science/dietarysurveys/dietsurveys/ (accessed February 2016).

18. McCullough ML, Feskanich D, Stampfer MJ, et al. (2002) Diet quality and major chronic disease risk in men and women: moving toward improved dietary guidance. Am J Clin Nutr 76, 1261-1271.

19. O'Donovan CB, Walsh MC, Nugent AP, et al. (2015) Use of metabotyping for the delivery of personalised nutrition. $\mathrm{Mol}$ Nutr Food Res 59, 377-385.

20. Li K, Brennan L, McNulty BA, et al. (2016) Plasma fatty acid patterns reflect dietary habits and metabolic health: a cross-sectional study. Mol Nutr Food Res 60, 2043-2052.

21. Expert Panel on Detection, Evaluation, and Treatment of High Blood Cholesterol in Adults (2001) Executive Summary of the Third Report of the National Cholesterol Education Program (NCEP) Expert Panel on Detection, Evaluation, and Treatment of High Blood Cholesterol in Adults (Adult Treatment Panel III). JAMA 285, 2486-2497.

22. Linseisen J, Kesse E, Slimani N, et al. (2002) Meat consumption in the European Prospective Investigation into Cancer and Nutrition (EPIC) cohorts: results from 24-hour dietary recalls. Public Health Nutr 5, 1243-1258.

23. Kaluza J, Åkesson A \& Wolk A (2014) Processed and unprocessed red meat consumption and risk of heart failure: prospective study of men. Circ Heart Fail 7, 552-557.

24. Kaluza J, Åkesson A \& Wolk A (2015) Long-term processed and unprocessed red meat consumption and risk of heart failure: a prospective cohort study of women. Int J Cardiol 193, 42-46.

25. Daniel CR, Cross AJ, Koebnick C, et al. (2011) Trends in meat consumption in the USA. Public Health Nutr 14, $575-583$.

26. Denova-Gutiérrez E, Tucker KL, Flores M, et al. (2016) Dietary patterns are associated with predicted cardiovascular disease risk in an urban Mexican adult population. J Nutr 146, 90-97.

27. European Food Safety Authority (EFSA) Panel on Dietetic Products Nutrition and Allergies (2010) Scientific Opinion on Dietary Reference Values for fats, including saturated fatty acids, polyunsaturated fatty acids, monounsaturated fatty acids, trans fatty acids and cholesterol. EFSA $J \mathbf{8}$, $1461-1568$.

28. Li K, McNulty BA, Tiernery AM, et al. (2016) Dietary fat intakes in Irish adults in 2011: how much has changed in 10 years? Br J Nutr 115, 1798-1809. 